Archives de sciences sociales des religions

141 | janvier-mars 2008

Mémoires

\title{
Le pentecôtisme brésilien au Cap-Vert
}

L'Église Universelle du Royaume de Dieu

Pierre-Joseph Laurent et Claudio Furtado

\section{CpenEdition}

\section{Journals}

Édition électronique

URL : https://journals.openedition.org/assr/12912

DOI : $10.4000 /$ assr. 12912

ISSN : $1777-5825$

Éditeur

Éditions de l'EHESS

Édition imprimée

Date de publication : 1 mars 2008

Pagination : 113-131

ISBN : 978-2-7132-2189-7

ISSN : 0335-5985

Référence électronique

Pierre-Joseph Laurent et Claudio Furtado, « Le pentecôtisme brésilien au Cap-Vert », Archives de sciences sociales des religions [En ligne], 141 | janvier-mars 2008, mis en ligne le 02 juillet 2011 , consulté le 09 juillet 2021. URL : http://journals.openedition.org/assr/12912 ; DOI : https://doi.org/ 10.4000/assr.12912 


\section{Pierre-Joseph Laurent, Claudio Furtado Le pentecôtisme brésilien au Cap-Vert L'Église Universelle du Royaume de Dieu ${ }^{1}$}

En contrebas du Plateau, quartier historique de Praia, la capitale, convergent quatre fois par jour près d'un millier de fidèles venus assister au Templo Maior ${ }^{2}$ à la «réunion » dédiée, selon les jours de la semaine, à la prospérité, la santé, la libération ou encore la purification. "Ce qui s'est passé dans la vie de cette personne peut se passer dans votre vie "; "Participez à une prière puissante et votre vie va changer ", ou encore : "O mes amis, votre vie peut changer. Il reste dix minutes pour vous rendre à la réunion, ne remettez pas à demain ce que vous pouvez faire aujourd'hui... » Dès l'aube, la très populaire « Radio Crioula » (inaugurée en 2004) et, depuis août 2007, les émissions religieuses produites par la chaîne de télévision de l'évêque Macedo, la Rede Record International, poussent les fidèles à rejoindre le temple de l'Église Universelle du Royaume de Dieu (EU). La radio et la télévision diffusent des témoignages de croyants issus des différents quartiers de la capitale ou des villages avoisinants de l'île de Santiago et miraculeusement exaucés par la puissance de l'Esprit Saint. Le pasteur-animateur, pour remercier l'interlocuteur de sa participation, lui propose de donner les noms de proches qu'il souhaiterait bénir. En fin d'émission, les auditeurs, après avoir été préalablement conviés à remplir un verre d'eau et à le poser à proximité du poste de radio ou de télévision, afin qu'elle soit, elle aussi, consacrée, attendent les conseils et la prière de puissance prononcée par l'évêque du Cap-Vert, le pasteur brésilien Marcelo Abrantes, figure charismatique et omniprésente de l'Église Universelle dans le pays depuis 1999.

Dès la découverte de l'archipel, au XV $\mathrm{XV}^{\mathrm{e}}$ siècle, par les navigateurs portugais, l'Église catholique, qui compte officiellement, aujourd'hui, plus de $93 \%{ }^{3}$ de

1. Les données de cet article ont été recueillies lors de cinq missions d'enquête dans l'archipel du Cap-Vert : ces missions ont été financées par le Fond National de la Recherche Scientifique de Belgique (FNRS - programme FRFC : juin 2004, mars 2005, septembre 2005, septembre 2007) et par l'Institut d'Études du Développement (IRD) en France, UR Construction identitaires et mondialisation dirigée par Marie-José Jolivet (décembre 2003).

2. Le Templo Maior de Praia a une capacité de mille six cents places assises.

3. Selon les statistiques nationales établies en 2006 (Instituto Nacional de Estatística do Cabo Verde). 
fidèles sur une population totale de près de 475000 habitants ${ }^{4}$, a joué un rôle majeur dans la constitution de la société et de l'identité capverdienne. Il est un fait que cette société créole s'est structurée sur certaines valeurs catholiques et que la population s'implique largement dans leurs formes les plus populaires, là où la vie est scandée par les festivités rendues en l'honneur des Saints, patrons et protecteurs, de chaque association, village, quartier ou ville. Au Cap-Vert, c'est assez logiquement que le catholicisme constitue la cible privilégiée de l'Église Universelle. De sorte que, tout en s'appuyant très largement sur ce fond culturel chrétien, elle peut en inverser les symboles. Ils deviennent alors la principale cause de la résignation et de l'échec individuel face à l'esprit de la pauvreté lorsqu'il est, par exemple, déclaré dans la cathédrale de la foi de Praia qu' « Accepter la Croix (la souffrance), n'est pas inscrit dans la Bible mais plutôt que la prospérité est un don de Dieu. " Dans ce contexte, le développement et le succès manifeste, au cours des dix dernières années, de l'Église Universelle, Église d'origine brésilienne qui articule de manière explicite Dieu et Argent, par l'adoption du trait principal de la théologie de la prospérité qui veut qu'un vrai croyant ne peut être pauvre, étonnent à première vue.

Ce texte montrera que l'Église Universelle s'établit avec un certain succès dans l'archipel du Cap-Vert, à la faveur d'un moment singulier où le pays connaît une importante période de croissance économique (6 à $7 \%$ par an depuis dix ans et une inflation maitrisée de l'ordre de 2 à $5 \%$ par an pour la même période $)^{5}$. Les indicateurs du PNUD positionnent le Cap-Vert au deuxième rang de l'Afrique après Maurice, une place qui ne traduit toutefois pas nécessairement une réduction de la précarité ni de la vulnérabilité alimentaire pour certains groupes de personnes résidant surtout dans les centres urbains en pleine expansion. L'embellie économique entraîne aussi une réforme importante de la culture et une augmentation des inégalités sociales que les autorités du pays peinent à réguler. La modernisation rapide du pays renvoie à un moment particulièrement instable. Les richesses se font et se défont, dans une micro société insulaire où pourtant les stratifications sociales, particulièrement marquées et solidement établies, limitent les chemins de l'ascension sociale et la redistribution des fruits de la croissance.

\section{L'Universelle dans le contexte capverdien}

L'indépendance fut tardive et l'archipel, qui connaît un climat aride et un environnement dégradé, hérita de l'économie de prédation coloniale. L'histoire de la traite - dans laquelle le Cap-Vert tiendra le rôle "d'îles entrepôts" (Carreira, 2000) en raison de sa position géographique - a laissé de profondes

4. Avec un taux de croissance démographique annuel de $1,9 \%$ (PNUD 2004) et (Instituto Nacional de Estat'stica do Cabo Verde).

5. FAO, rapport spécial Cap-Vert 2005. 
traces dans l'identité capverdienne. Mais la mémoire collective du pays s'établit aussi sur le souvenir de famines : douze au XIX siècle et huit au XXe siècle, jusqu'à l'indépendance en 1975. La dernière grande famine, entre 1940 et 1950, fit plus de trente mille victimes sur une population totale d'environ cent cinquante mille personnes (Carreira, 2000 : 208 ; Silva Andrade, 1996 : 151 ; 2000). C'est à la lumière de ces informations qu'il convient d'apprécier la valeur du réel développement économique que le pays a su susciter et piloter depuis l'indépendance : le PIB passera de 190 dollars par habitant en 1975, à 1938 en 2006, avec une espérance de vie de 71,2 ans (FMI, 2006). Fort de ces bons indicateurs économiques, couplés à ceux du développement du capital humain, le pays est devenu en janvier 2008, un Pays de Développement Moyen (PDM), selon les statistiques établies par le PNUD.

Ceci appelle cependant une analyse plus détaillée afin de préciser le contexte singulier dans lequel évolue l'Universelle au Cap-Vert. Ainsi, pour le PIB, le secteur des services compte pour près de $72 \%$ de la richesse nationale, alors que $17 \%$ sont attribués à l'industrie et la construction, pour seulement $11 \%$ à l'agriculture et la pêche. Pour apprécier cette répartition, relevons qu'une partie des bons résultats sont dus aux transferts financiers des émigrés et à l'aide publique au développement qui représentent ensemble plus de $24 \%$ du PIB. De plus, le développement rapide, en une décennie, du secteur touristique, dans les îles de Sal et de Boa-Vista, qui représente aujourd'hui $10 \%$ du PIB, repose essentiellement sur des investisseurs étrangers (Italie, Portugal...) (Rocha Costa, 2003).

De ceci, établissons que le bien-être ne touche pas tous les Capverdiens. En 2004, le taux de pauvreté était estimé par le PNUD à 36,7\% et celui de pauvreté extrême à $19,7 \%$, soit au total $56,4 \%$ de la population (PNUD, 2005). Dès lors, la répartition des fruits de cette croissance devient un enjeu déterminant de la cohésion de la société capverdienne et un réel défi politique. L'indice de Gini, qui apprécie le degré de concentration des inégalités (en termes de revenu) au sein d'une population donnée, connaît en une décennie une véritable explosion pour reprendre les termes du ministère des Finances. Cet indice qui varie de zéro, pour une société la plus égalitaire, à un, pour la plus inégalitaire, est passé de 0,43 en 1988-1989 à 0,59 en 2001-2002; ce qui met le Cap-Vert au même niveau que le Brésil ; seuls l'Afrique du Sud $(0,593)$, le Nicaragua $(0,603)$ et le Swaziland $(0,609)$ connaissent un taux plus élevé ${ }^{6}$. Si l'archipel rejoint les Pays de Développement Moyen, il s'apparente aussi aux pays les plus inégaux en termes de revenu, tel le Brésil dont l'influence culturelle est manifeste. Il est intéressant de relever que l'Église Universelle s'implante avec un certain succès plutôt dans des pays qui connaissent une croissance économique notoire couplée

6. Documento de estratégia de crescimento e de redução da pobreza, Republica de Cabo Verde, Ministério das Finanças e do Planeamento, setembro de 2004, p. 35, note 40 ; PNUD, Rapport mondial sur le développement humain, 2001, annexes, pp. 182-185. 
à l'accroissement des inégalités (Afrique du Sud, Angola, ou encore Brésil, Paraguay, voire Portugal).

Il est un fait que ces changements économiques entraînent de profonds bouleversements culturels et faisons l'hypothèse que l'Église Universelle a su s'en nourrir pour développer une offre religieuse capable de prendre en considération les conséquences de cette situation sur les populations. Ricardo Mariano montre que pour le Brésil, "l'EU a su utiliser et exploiter à son avantage le contexte économique, culturel, politique et religieux. Et notamment les crises sociales et économiques, l'augmentation du chômage, la croissance de la violence et de la criminalité (...), l'ouverture politique et la diffusion des moyens de communication de masse » (Mariano, 2004 : 122). Pour de nombreuses familles capverdiennes, cette situation se traduit, en guise de survie, par l'attente des mandats envoyés par un parent émigré. En raison des départs habituels des hommes, parfois pour de nombreuses années (et parfois sans retour), $40 \%$ des familles sont dirigées par une femme ${ }^{7}$. En milieu rural, cette situation conduit nombre de femmes à devenir chefs d'exploitation sur des terres souvent louées qu'elles entretiennent avec leurs enfants (Furtado, 1993). Malgré l'importance du catholicisme, le mariage reste peu valorisé $\left(16,5 \%{ }^{8}\right)$. Et dans la majorité des ménages, le couple ne cohabite pas. Les réunions de l'Universelle ne manquent jamais, en fin de culte, d'évoquer cette situation : les fidèles, sans nouvelles de parents à l'étranger, sont invités à apporter les photographies des proches perdus de vue, et les brandissant au-dessus de leurs têtes, le pasteur demande alors à Dieu de rétablir le lien. De nombreux témoignages portent précisément sur la reconstruction, «miraculeuse ", de ces liens familiaux. Dans ce contexte, la figure du migrant devient un modèle. La jeunesse est fascinée par les styles de vie venus d'Europe, des États-Unis ou du Brésil que les migrants arborent de manière ostentatoire lors de leurs congés. Pendant quelques semaines, ils flambent leur vie au prorata des sacrifices qu'ils endurent au loin. Par-delà cette vitrine éphémère où le migrant entretient une identité de gagnant vis-à-vis de ceux restés au pays, à leurs contacts, la jeunesse capverdienne entretient l'espoir d'un départ et d'une ascension sociale rapide.

Résumons-nous, un ensemble de facteurs inter-reliés conduit à cette situation : la croissance économique, l'augmentation du taux de la pauvreté, le chômage, l'oisiveté, le désir d'accéder à la consommation, l'instabilité familiale, l'occurrence de la famille matricentrée associée à la figure absente du père, une certaine prostitution domestique, la présence de l'alcoolisme et de drogues, la délinquance et la criminalité... Ceci limite la marge de manœuvre de certains groupes de population ${ }^{9}$, parmi lesquels les difficultés de la survie peuvent éroder

7. Institut National de Statistique, censo 2000, p. 59.

8. Ibid., p. 60.

9. Dans le sens ici d'Amartya Sen qui a associé l'idée de liberté à celle de choix de vie d'une part, à celle de responsabilité collective d'autre part. L'enjeu est celui d'une justice sociale centrée 
les valeurs de la société et valoriser des comportements de débrouille, liés au cumul des échecs et à la mésestime de soi. C'est en quelque sorte ce paradoxe qu'à bien montré Karl Polanyi dans sa célèbre étude, où l'entrée de la société dans l'économie de marché se traduit, pour certains groupes de population, par la dislocation des modes de vie (soit des principes d'échange : réciprocité, redistribution, gestion domestique). Il s'agit d'une vie dans un vide culturelle. Cette «catastrophe culturelle », pour reprendre la formule de l'auteur, favorise la dégradation humaine et le développement de "l'homme sans maitre ». Cette situation conduit à la dépendance, à la fois par l'aide qui s'oppose à la liberté de choisir son mode de vie et par la déliquescence des principes du vivre ensemble (Polanyi, 1993 : 212-213). C'est précisément dans un contexte de bouleversements culturels et donc des identités sociales, ainsi que du changement des mentalités, que l'Église Universelle va s'implanter au Cap-Vert.

\section{Aspects organisationnels}

Ses débuts y furent difficiles. Bina, une «ouvrière » de la première heure, raconte qu'ils n'étaient qu'une dizaine à se réunir dans une petite salle de la capitale. Devant ces difficultés, le Pasteur Alexandre qui, du Portugal, débarque à Praia en 1992, décide de partir à Mindelo sur l'île de São Vicente où il installe le premier siège ${ }^{10}$. C'est à partir de cette ville portuaire qu'il revient à la capitale (1994) et parvient progressivement à établir l'Église sur l'ensemble de l'archipel. Elle compte à ce jour une vingtaine de lieux de culte, plus communément appelés Centre d'Aide Spirituelle. Il quitte le Cap-Vert en 1999 pour São Tomé où il meurt.

Le pasteur Marcelo Abrantes, actuel leader de l'Église Universelle au CapVert, réside à Paria. Il possède le titre d'Évêque (bispo) et se trouve à la tête d'une région qui comprend le Cap-Vert, São Tome e Principe et la Guinée-Bissau. Originaire de l'État de Para au Brésil, il est issu d'une famille modeste et aurait rencontré l'Universelle dès ses débuts, soit à la fin des années $1970^{11}$. Il arrive au Cap-Vert en 1999 après avoir participé à l'installation de l'Église brésilienne au Portugal. La première église est implantée à Johannesburg en Afrique du Sud en 1992. Quasi simultanément, elle s'établit par affinité linguistique au Mozambique, en Angola et en Guinée-Bissau. Elle est aujourd'hui présente dans de nombreux pays et les communautés les plus fortes, en dehors de la zone lusophone, se trouvent

sur l'idée de "droits à certaines capacités " (1993). À ce propos, voir aussi Ricoeur (2004); cette définition va dans le même sens que celle de la politique des Fronts à Haute Intensité de Main-d'œuvre (FAIMO) du Cap-Vert qui consiste précisément à compléter les revenus des populations rurales.

10. Voir le cas du cinéma Miramar de Mindelo dans lequel l'EU avait initialement installé son lieu de "réunion".

11. Entretien réalisé en juin 2004 à Praia. 
au Botswana, Congo, Côte-d'Ivoire (Mary, 2002), Kenya, Lesotho, Malawi, Ouganda, Zambie, Zimbabwe ${ }^{12}$.

Pour mieux cerner les éléments qui conduisent l'Église Universelle à débarquer dans l'archipel, rappelons qu'elle fut fondée par Edir Macedo à Rio de Janeiro en 1977 (Almeida, 1996 ; Campos, 1999 ; Mariano, 1995 ; Oro, 2005), et que, selon Paul Feston, elle sortit des frontières brésiliennes dès 1985 pour s'établir au Paraguay (Freston, 1999, 2001). Ce fut, toutefois, l'investissement dans des réseaux de radios et l'acquisition en 1989 de la télévision Rede Record qui marqua un tournant dans l'expansion (Campos, 1999 : 363), rejoignant en cela les pratiques des télévangélistes nord-américains et de leurs " églises électroniques ». Aujourd'hui, au Brésil, où la télévision tient un rôle important dans la société, l'Église Universelle est, selon Campos, une véritable entreprise religieuse (1997, 1999) qui occupe le plus grand espace télévisuel du pays (Mariano, 2004 : 122). C'est en 1990 qu'elle entre aux États-Unis, en Argentine et au Portugal. Le continent africain sera abordé en 1992 par deux voies : celle de l'Afrique du Sud où, dès le milieu des années 1990, Macedo, considérant ce pays comme la porte d'entrée du continent, installe une résidence au Cap qu'il partage avec New York (Freston, 1999 : 388); celle du Portugal ensuite, où l'Église s'installe en 1989, et à partir duquel l'entreprise religieuse organisera son expansion dans les pays lusophones d'Afrique ${ }^{13}$.

À Praia, la nuit, une enseigne lumineuse au logo de l'Église - une colombe blanche sur un cœur rouge - rappelle aux citadins le Templo Maior inauguré en octobre 2003. Ce vaste bâtiment en béton, peint en blanc, ressemblait jusque récemment à un hangar en construction. L'achèvement de l'édifice en fait le plus vaste bâtiment religieux du pays. Durant quatre ans, les réunions se sont déroulées au rez-de-chaussée dans une salle inconfortable. Début 2007, un second étage, richement aménagé, accueille la nouvelle salle de culte. On y accède par un large double escalier qui s'ouvre sur une grande salle de forme elliptique. La couleur blanche domine. Seuls les vitraux latéraux et ceux situés derrière l'estrade tranchent de leurs couleurs vives. La salle est climatisée et la lumière artificielle est intégrée dans le plafond, ainsi que la sonorisation qui permet de suivre le culte dans de bonnes conditions. Les fauteuils en bois, recouvert de simili cuir, sont confortables. Reliés les uns aux autres, les sièges sont disposés en petites rangées alignées entre les nombreuses travées qui rayonnent vers l'estrade surélevée de près d'un mètre par rapport à l'assemblée, disposition qui assure une bonne visibilité et positionne le pasteur, tel l'artiste, face à son public. Le mobilier de culte est assez sommaire. Il se compose d'un autel, de fauteuils et des chaises et au centre de la scène, le pupitre, derrière lequel se tient le pasteur animateur de la réunion. La sonorisation, l'orchestre et le matériel audio-visuel

12. À ce propos, voir les articles de Marion Aubrée $(2001$; 2003).

13. Selon L.S. Campos, 36,5\% des temples de l'EU sont installés en Afrique lusophone et 32,6\% dans la seule Afrique du Sud (1999: 365). 
complètent le mobilier de culte et sont disposés de manière ostentatoire. La salle se veut luxueuse. Sa réalisation est censée attester autant de la générosité des fidèles que de l'intervention divine. Ce faste, qui me fut longuement commenté par les croyants, notamment la qualité des fauteuils et de la climatisation, participe aussi de l'image de "gagnant » et de respectabilité que veut attribuer l'Église Universelle à ses fidèles, à qui le beau, la richesse et le bonheur sont promis.

Créée en 2000, la Rede Record Internacional présente un ensemble complet d'émissions. La chaîne émet par satellite aux États-Unis, au Canada, en Europe et sur le continent africain, avec une liaison particulière avec les pays lusophones. Et au Cap-Vert, pays où la télévision occupe une place de choix dans les foyers, la Rede Record vient de négocier sa présence avec l'octroi, par le gouvernement, en janvier 2007, d'une licence pour émettre sur l'ensemble du territoire. Les premières émissions religieuses, filmées directement au Templo Maior de Praia, ont débuté en août 2007. Sur cette chaîne, et prisées des capverdiens, les telenovales brésiliennes - longues séries qui, telles des sagas, mettent en scène les relations entre maîtres et esclaves, la rigidité de la «bourgeoisie du café paulista » du début $\mathrm{du} \mathrm{XX}^{\mathrm{e}}$ siècle face à la sensibilité populaire, ou encore, les caprices d'une belle du peuple qui, dans le Brésil d'aujourd'hui, se joue d'un jeune riche côtoient les émissions religieuses produites par l'Église. Il y est notamment question de témoignages de croyants capverdiens miraculeusement guéris de leurs souffrances. Le succès médiatique est incontestable. Par ces émissions, les témoins, croyants souffrants, miraculeusement guéris par l'intervention divine, connaissent pour un moment, la gloire d'un personnage médiatique. Reconnu par ses voisins et amis, le témoin est félicité pour sa prestation qu'il commente alors inlassablement lorsque l'occasion s'en présente.

Notons que le témoignage renvoie à un rapport de proximité, de familiarité, de voisinage : c'est mon voisin de quartier qui hier à telle heure a bénéficié d'un miracle dans sa vie, alors pourquoi pas moi ? Les témoignages sont sollicités durant les " réunions " après le rituel d'exorcisme. Il constitue la "scène " généralement sélectionnée pour être diffusée lors d'émissions de Radio créole ou de la Rede Record. Une vingtaine de personnes sont sélectionnées. C’est une occasion, pour elles, de monter sur la scène et de s'adresser à l'assemblée. Il est fréquent que les témoins prennent un vrai plaisir à se trouver ainsi sous les feux de la rampe et cherchent à faire rire. Les interventions sont dirigées par le pasteur qui pose les questions : "Quel est votre nom ? Vous habitez où ? Et alors, qu'est-ce qu'il est arrivé dans votre vie, mon ami(e) ? » Et le témoin de répondre sur un mode très standardisé : "J'avais trois ans lorsque mon père a émigré au ÉtatsUnis. Depuis, il ne nous a jamais donné de nouvelles, ni envoyé aucun mandat. J'ai maintenant trente ans. Il y a deux mois que j'ai commencé à fréquenter l'EU et il y a deux semaines mon père a pris contact avec moi ". L'animateur : "Vous entendez ? C'est fantastique. Vous voyez la puissance de Dieu, c'est un miracle... Applaudissons. Et remercions Dieu pour sa grâce ». 
Tout est organisé pour favoriser le culte de la personnalité qui joue un rôle important dans la scénographie de l'Universelle. Impeccablement vêtu d'un costume tendance, l'accent brésilien prononcé, le pasteur titulaire de Praia, le bispo (évêque) Marcelo, comme dans les séquences quotidiennes des feuilletons brésiliens (telenovelas), incarne la figure de l'entrepreneur dynamique, capitaine d'une entreprise religieuse florissante. Riche et séduisant, tout semble lui réussir. L'identité proposée aux fidèles est bien celle de ce pasteur - entrepreneur, véritable emblème d'une ascension sociale aboutie et d'une vie heureuse.

Selon le bispo Marcelo, l'Église Universelle compte près de vingt mille fidèles pratiquant quotidiennement dans le pays (soit une estimation de $4 \%$ de la population) ${ }^{14}$, dont près de trois mille cinq cents pour la ville de Praia (qui compte aujourd'hui plus de cent mille habitants). Il y aurait dans le pays une quarantaine de pasteurs, rapidement formés aux pratiques de l'Église. Le Templo Maior de Praia reste ouvert en permanence et les pasteurs sont employés à temps plein, c'est-à-dire qu'ils n'ont pas d'autres activités comme c'est souvent le cas pour les pasteurs des autres Églises évangéliques. Pour ce lieu de culte de la capitale, les pasteurs, outre l'aide des pasteurs auxiliaires, peuvent compter sur l'appui efficace et dévoué d'une centaine d'ouvrier(e)s qui arpentent les quartiers de la ville et encadrent avec zèle les réunions quotidiennes.

Le management de l'Église Universelle est particulièrement spécialisé, agressif, dynamique et efficace. Il repose sur le principe qu'il faut aller chercher les adeptes potentiels là où ils se trouvent: dans les quartiers populaires des villes ${ }^{15}$. Pour cela l'Église peut compter sur la radio et, depuis peu, la télévision, mais également sur le travail des nombreux fidèles "ouvrier(e)s " pour attirer les fidèles au temple. Le management professe qu'une fois franchi le seuil du temple, il ne faut plus laisser en paix les nouveaux venus. Pour cela, il faut sans cesse surprendre, changer, bousculer, convaincre et gérer l'espoir. Dans la hiérarchie, les ouvriers et ouvrières se situent à une charnière de l'édifice institutionnel du fonctionnement d'un temple. Ces fidèles peuvent espérer une ascension sociale à l'intérieur même de l'Église. Cette situation singulière fait d'eux, parmi les fidèles, la catégorie la plus exploitée par l'institution. En effet, si certains croyants viennent chercher au temple une solution concrète à leurs problèmes et en restent là, d'autres, devenus ouvriers, cherchent à s'impliquer de plus en plus dans l'Église avec l'espoir d'être finalement repérés par le pasteur, cherchant ainsi, après avoir témoigné parfois de nombreuses années de bons et loyaux services envers le "pasteur patron ", à passer dans la catégorie des pasteurs auxiliaires, voire des pasteurs

14. Cette manière de compter les adeptes est propre à l'EU qui propose, tous les jours de la semaine, une réunion spécifique à différentes heures de la journée. Sur la base d'observations étendues sur plusieurs missions réparties sur trois ans, il semble que ce chiffre soit surestimé.

15. Sur l'agressivité des campagnes de l'EU (par exemple envers les autres cultes), ainsi que sur les nombreuses polémiques qu'elles suscitent concernant notamment l'enrichissement de ses responsables, voir Giumbelli $(2002,456)$. 
avec, alors, la chance d'accéder à un emploi et à un revenu. Mariano a montré que, pour s'élever dans la hiérarchie ecclésiastique de l'Église Universelle, les candidats doivent faire preuve de leur capacité à collecter la dîme et les offrandes. Il a existé une faculté de théologie de l'Église Universelle, rapidement fermée lorsque Macedo s'est rendu compte que, pour atteindre ses objectifs, la théologie était inutile, voire même éloignait les pasteurs des objectifs de base. Un bon pasteur doit se mesurer en termes "de numériques" et de financement pour l’Église (Mariano, 2004 : 126).

Signalons qu'en dehors de l'Église catholique et de l'Universelle, Praia compte une dizaine de dénominations pentecôtistes, ainsi que deux Églises indépendantes originaires du Ghana et du Nigeria. L'Église des Assemblées de Dieu, numériquement la plus importante du monde, a été introduite en 1986 (affiliation aux Assemblées de Dieu en 1990) par le pasteur capverdien P. Jorges Fernandes alors résidant à Dakar. Les autres mouvements religieux sont : l'islam introduit par des migrants africains (sénégalais surtout) et qui, selon l'Église catholique, représenterait près de $3 \%$; l'Igreja do Nazareno (Église baptiste), introduite en 1907 par le pasteur Breese, ancien migrant capverdien aux États-Unis, avec six à sept mille fidèles; l'Igreja Baptista, érigée en 1960, d'une dissidence de la précédente, par un migrant capverdien aux États-Unis ; il existe aujourd'hui au Cap-Vert d'autres Églises baptistes, indépendantes ou fédérées au sein d'associations : l'Église Adventiste du Septième jour, introduite en 1934 par un migrant de retour des États-Unis; les Témoins de Jehovah introduits en 1958 à partir des États-Unis; depuis 1986, les Mormons, et le Rationalisme chrétien (mouvement spirite).

\section{La " théologie de la prospérité " au Cap-Vert}

La conjoncture décrite ci-dessus brouille les anciens repères de l'identité capverdienne et altère le patrimoine organisationnel de la société. Certains groupes de population, confrontés aux mutations rapides de la société, se résignent et parfois peuvent être tentés par la violence, l'alcool, la drogue, voire la prostitution... L'Église Universelle se montre particulièrement attentive aux expressions multiformes des souffrances sociétales contre lesquelles elle met tout en œuvre pour offrir aux fidèles la possibilité de se recomposer une identité positive plus en accord avec la société capverdienne en pleine transformation.

C'est dans ce contexte que s'implante au Cap-Vert, avec un certain succès, cette Église qui articule sans ambages Dieu et Argent, et qui veut qu'un vrai croyant ne soit pas pauvre. Elle adopte ainsi la caractéristique principale de la théologie de la prospérité où avoir la foi attribue au croyant le pouvoir d'exiger de Dieu, voire d'être dans la position de s'approprier, l'autorité divine pour faire exister les choses qui n'existent pas. C'est dans ce sens que plusieurs auteurs parlent de «confession positive» (Mariano, 2004 : 201). Selon les principes de 
la théologie de la prospérité, les chrétiens ont non seulement été délivrés du péché originel par le sacrifice de Jésus sur la croix, mais ont, en outre, acquis le doit, hic et nunc, à une vie bonne, prospère car libérée de la souffrance et des ruses du diable. Ce qui est fondamental dans la doctrine de l'Église, c'est que Dieu n'a pas seulement promis ce droit à la prospérité aux croyants, mais que ce droit leur a déjà été donné au plan spirituel. En conséquence, les rituels visent à permettre aux fidèles de s'emparer de ces biens ${ }^{16}$.

Analysons la manière dont ces principes de la "théologie de la prospérité » prennent corps au Cap-Vert. Sur les ondes de la radio et de la télévision, les animateurs martèlent qu'ils s'adressent à "ceux qui souffrent ", croyants ou non, et plus particulièrement à «ceux qui se débattent quotidiennement avec des problèmes financiers, des violences conjugales, la drogue, la maladie, les insomnies, le mauvais œil, la jalousie, ou encore, ceux qui pensent au suicide, qui sont sans nouvelles d'un fils, d'une fille, d'un père ou d'une mère immigrés, ou enfin celles qui ne peuvent pas avoir d'enfant "; ils définissent ces groupes divers auxquels ils destinent prioritairement leurs activités comme «ceux qui sont fragiles ", "ceux qui veulent changer leur vie » ou encore qui « veulent la prospérité ».

Dans le contexte capverdien, nos informateurs résument l'objectif de l'Église Universelle comme étant celui de "renforcer les gens qui sont fragiles ", pour qu'ils « puissent faire l'expérience de la puissance de Dieu agissant sur eux, afin qu'ils en sortent changés ». Remarquons que la catégorie de " ceux qui souffrent » est sémantiquement plus étendue que celle "de pauvre "; la catégorie "ceux qui souffrent" renvoie à ce contexte d'incertitude qui insécurise ${ }^{17}$. Selon la rhétorique de l'Universelle «ceux qui souffrent » sont plus particulièrement: " celui à qui le médecin a dit qu'il ne pouvait plus rien faire, celui qui veut se libérer de l'alcool ou de la drogue, celui qui veut connaitre la paix dans son couple, celui qui veut que sa vie financière change...» Il est question d'une expérience personnelle, individuelle, où le corps devient le théâtre et le lieu de la souffrance qui se résume, le plus souvent, à un conflit intérieur sans issue : « je suis nerveuse, j'ai mal la tête, ma maison est devenue une pharmacie... " Alors que la vie semble vide de sens car envahie d'hésitations, l'Église Universelle propose une explication simple et efficace. Le vide évoque l'inaction et la résignation devant la souffrance. La dépendance est identifiée aux vices, c'est-à-dire au vide censé conduire à l'alcoolisme, à la prostitution, à la violence, à la délinquance.

Dépasser ce constat repose sur la conviction qu'il convient de changer d'attitude pour changer de vie, en intériorisant l'idée que ce projet est non seulement

16. Pour plus d'informations concernant la théologie de la prospérité, d'origine nordaméricaine, dès 1950, et liée au télévangélisme voir Martin (1990), et pour celle développée par l'EU : Campos (1997 : 367-378).

17. Cf. la notion de "modernité insécurisée » (Anxiety-Producing Modernity) (Laurent, 2005). 
possible mais accessible à tous. Ainsi ce pasteur qui, lors d'une cérémonie en septembre 2007 dans la cathédrale de Praia, expliquait : « La manière dont vous voyez Jésus, est la manière dont Jésus fera des miracles dans votre vie ou n'en fera pas (...) Si vous voyez Jésus comme celui qui faisait des miracles, alors il ne se passera rien. Par contre, si vous le voyez comme celui qui n'a pas changé et qui a toujours le pouvoir de libérer du mal et de changer la vie des gens alors vous pouvez vous transformer. " Pour l'Universelle, la sortie de la résignation, entretenue selon eux par les catholiques, serait la clé du succès: "Jésus n'a jamais dit que le pauvre doit rester pauvre ou que vous devez accepter votre croix. Il est écrit dans la Bible que le pauvre doit, par la foi, être riche. »Et de préciser qu'il est "erroné de s'en remettre à la volonté de Dieu. Changez d'attitude, ayez la certitude que Dieu veut votre bonheur ».

Les notions de malheur, de fragilité, de souffrance sont au cœur de la représentation que les fidèles se font de leur situation ; l'Église Universelle les conduit à les appréhender comme une imposture (Fiallho-Costa, 2002: 81). La souffrance équivaut à un mauvais esprit, voire au démon qui symbolise la jalousie, la violence, la pauvreté, le désir, la maladie, dont il convient de se débarrasser (Birman, 1997, 1998 ; Campos, 2003 ; Gomez, 1993). Pour Bina, une "ouvrière " du Templo Maior, "le démon est une personne qui vit avec les gens et leur apporte le malheur ». Ce que les fidèles viennent chercher, c'est l'espoir d'être libre et de trouver la force pour s'extraire du poids de la culpabilité, transmise, selon les pasteurs, par l'Église catholique. La souffrance devient un scandale, un esclavage, dont le fidèle cherche à tout prix à se dégager (Pimentel, 2005). Et le mal (fatalité, malheur, pauvreté, souffrance) est ce dont il faut libérer son corps, pour se libérer soi-même. Une singularité de l'Universelle consiste à intérioriser ce mal-être, à éviter de le porter sur l'espace public ${ }^{18}$. La victoire est une lutte individuelle. La souffrance ne renvoie pas à une analyse en termes politiques. La seule dimension collective, lorsqu'elle existe, se rencontre quand, au temple, debout, serrés les uns contre les autres, les fidèles captent, les mains tournées vers le Ciel, la puissance de Dieu. Ici le corps circonscrit le conflit. Il est le réceptacle du mal-être sociétal et le théâtre de l'affrontement du fidèle avec lui-même. La vie vide, faite d'hésitations et de résignations, équivaut au mal symbolisé par les actions des mauvais esprits qui prennent sens dans le corps dévoré, possédé. La « logique corporelle », pour reprendre la formule d'André Mary (2000: 186-187), met en relation la composition de la personne humaine (l'identité personnelle) et le corps pensé comme le lieu où s'inscrivent les marques de "l'agression sorcière ", celle de l'ennemi, ou encore de cet autre qui vous veut du mal. Dans la rhétorique de l'Église Universelle du Cap-Vert, l'ennemi est anonyme et impersonnel, même lorsqu'il désigne l'autre, comme dans le cas du mauvais œil ou de la jalousie.

18. À la différence de la théologie de la libération au Brésil. À ce propos, voir Corten, notamment le chap. 7 : La secte : l'anti-politique du pauvre (1995). 
Dans ce cas, celui qui vous veut du mal est identifié à une part maudite de soimême. De cette conception du malheur, il est intéressant de relever la différence par rapport aux « maladies indexées » de la majorité des pentecôtistes du continent africain (Laurent, 2003).

La victoire (sur soi-même) s'établit en deux étapes. La première renvoie au combat avec un ennemi intérieur identifié, personnifié : un mauvais esprit. Ce combat est ritualisé dans l'exorcisme. Il symbolise, d'abord, l'identification concrète du mal et, ensuite, la rémission par l'extraction de l'entité maligne (fatalité, souffrance, pauvreté) logée dans le corps. L'exorcisme est le traitement spirituel ad hoc: "le démon doit sortir des corps car il bloque le chemin de la victoire ". L'ennemi est ainsi identifié : le démon est cet autre qui vous persécute. Et, par sauts analogiques, cet autre est finalement assimilé au doute qui vous ronge, ou encore, à votre propre attentisme face à votre situation.

Le second temps est celui de la "définition ", c'est-à-dire de la certitude qui devra progressivement s'installer pour faire naître la « victoire ». Cette certitude repose sur l'idée que le mal, c'est moins l'autre qui vous persécute (comme c'est généralement le cas en Afrique continentale) que l'autre de soi-même, la part mésestimée de soi qui inhibe toute action: "si vous voulez être heureux, c'est vous qui décidez! » ou encore "La personne qui connaît sa vérité se libère ! ", entend-t-on lors des réunions.

Le croyant n'est pas né pour souffrir, mais sa vie reflète ses propres attitudes. Il est en outre précisé que la volonté de Dieu voudrait que le fidèle ne reste pas dans le malheur ou au bas de l'échelle sociale. Ce dernier est donc convié à prendre possession de lui-même, à devenir responsable et maître de son destin, afin de s'émanciper de l'envie, la jalousie, la colère, l'alcool, la drogue, la nervosité, les hésitations, l'attentisme qui auraient envahi son existence. Se libérer du mal dépend uniquement de la personne, coupable de se complaire dans la résignation. Au regard du catholicisme, c'est le sentiment de culpabilité face au péché qui se déplace : tu n'es plus coupable face à Dieu, mais bien coupable face à toi-même.

Les formules assenées durant les "réunions ", du genre "sortez de la dépendance ", " conquérez une nouvelle vie ", "prenez attitude ", "parvenez à vous définir " équivalent à des principes pédagogiques qui visent la constitution d'une nouvelle identité positive. Ces principes peuvent se définir comme une forme d'éducation à la maîtrise de soi : "si vous voulez vraiment vaincre afin que votre nouvelle vie puisse se concrétiser, vous devez commencer par vous-même. Travaillez à votre propre révolte car la foi l'exige ». Ces principes d'éducation sont clairement mis en évidence lors de la campagne d'avril 2006 où ceux qui rencontrent des problèmes « pour obtenir un prêt bancaire, qui sont sans emploi ou qui ne parviennent pas à terminer leur maison... ", sont invités à conquérir la prospérité. Mais pour cela, par-delà la délivrance des corps possédés par l'intervention de la puissance divine, l'Église Universelle propose aux fidèles de suivre un itinéraire long et clairement balisé, dont la description informe sur sa 
stratégie : 1 - «la manière dont vous voyez Jésus est la façon dont il agira dans votre vie », mais ; 2 - « il faut se battre» et alors ; 3 - « votre vie va changer »; 4 - toutefois pour atteindre ce changement, il convient de se repentir, se convertir, être baptisé et valoriser la famille... Nous pourrions en rester là et prendre acte que ce processus pédagogique qui procure de l'énergie à ceux qui souffrent, les pousse à entreprendre et à tenter de (re)conquérir une place dans le Cap-Vert d'aujourd'hui. Mais avec l'Église Universelle, dans le même élan, le fidèle apprendra vite que, pour sortir de sa situation, il y a un prix à payer qui consiste, selon Edir Macedo lui-même, à « être disposé à accepter la responsabilité d'être un des associés et des administrateurs de l'œuvre de Dieu » (Macedo, 2000 ; Mariano, $2004: 20)$.

\section{Ambiguité de "l'entreprise religieuse "}

Le sacrifice de l'ancienne vie signifie le fait d'oser lâcher les fausses certitudes; il évoque aussi la difficulté d'avoir eu en conséquence à "se définir " un autre projet de vie, acceptable, et à acquérir la certitude de pouvoir le conduire à son terme alors que les difficultés antérieures inhibent tous les désirs d'entreprendre. La certitude, c'est la foi qui permet de conquérir un espace de liberté ${ }^{19}$ par l'adhésion à un cadre normatif contraignant, exigeant. Une liberté dans la contrainte donc, qui nécessite, tant cette reconstruction identitaire est exigeante, une alliance avec Dieu afin de pouvoir compter sur sa puissance dans les moments de doute. Pour le fidèle, la foi représente donc ce lien indispensable s'il veut renaitre.

Ce principe qui consiste à faire accompagner de la puissance divine le processus de renaissance à une autre vie résume toute l'ambiguité sémantique de l'Église Universelle. À la différence des fidèles qui escomptent une intervention miraculeuse, la conception des animateurs est plus prosaïque, voire ambiguë, lorsqu'ils affirment que Dieu exaucera les vœux les plus chers des croyants, mais qu'en même temps tout vient de la volonté des individus : "Oser la foi pour parvenir à atteindre le miracle. La victoire dépend seulement de vous ", ou encore, "Faites votre partie et Dieu fera la sienne. Ne vous résignez pas, ne portez pas votre fardeau. Sortez de votre condition et, si vous avez la foi, vous y parviendrez. Vos problèmes - mariage, santé, drogue, vices - c'est vous qui pouvez les vaincre car Dieu va vous donner la victoire. La lutte sera dure, mais la victoire est certaine. Le miracle arrive là où il n'y a plus de doute ». Il est clair que c'est moins Dieu que le fidèle lui-même qui a la possibilité de faire advenir le miracle : parvenir à une vie meilleure par l'adhésion à des principes normatifs nouveaux. Mais le point de vue doctrinaire est autre. Et c'est précisément ce

19. Voir à ce propos Michel de Certeau pour qui la maîtrise en propre d'un espace est, avant tout, le fait d'abandonner les tactiques et les ruses, soit aussi l'ambiguité et les hésitations (1994) ; (Laurent, 1998 ; Latouche, Laurent, Singleton, Servais, 2004). 
langage ambivalent qui institue l'Universelle en intermédiaire entre Dieu et les hommes, tout en permettant à l'Église de se dédouaner si le croyant n'atteint pas la situation qu'il désire.

L'Église Universelle s'institue en intermédiaire entre Dieu et les croyants lorsqu'elle se donne comme mission de faire savoir à Dieu la valeur du choix du fidèle : une vie en accord avec les préceptes de la Bible. Mais, dans un même temps, comme pour se protéger d'un éventuel échec, elle ne dit rien d'autre à ses fidèles que cet oxymore "le miracle consiste à acquérir la foi en soi ». Une foi qui permet d'entreprendre à la faveur d'une victoire sur ses propres peurs. Ce rôle d'intermédiaire tenu par les pasteurs de l'Universelle, se donne plus particulièrement à voir pendant certaines campagnes comme celles du "Feu Sacré » ou celle de la "Montée au Mont Sinaï », etc., qui peuvent être organisées selon un calendrier bien établi et plusieurs fois par an, telle celle que nous avons pu suivre et qui s'est déroulée à Praia durant quatre semaines en juin 2004. Les principaux éléments en sont : le vœu censé exprimer la quintessence de la nouvelle vie préalablement identifiée par le fidèle : «Avez-vous désigné (choisi, déterminé) votre désir (votre vœu) le plus important ? Alors pensez au sacrifice que vous allez faire ! "; la privation, étape déterminante, ne se fait pas par la prière, mais plutôt par des dons d'argent parfois importants - au prorata de la situation financière de chaque fidèle poussé à en déterminer lui-même le montant - qui visent à attirer l'attention de Dieu sur sa propre révolte, face à une vie anéantie par le malheur, la souffrance ou la misère. Dans ce système de pensée, Dieu, témoin de cet acte de foi, est en quelque sorte obligé de faire un geste : "En donnant tout, vous accédez à un droit sur Dieu. Dieu a votre sacrifice sur sa conscience. C'est obligé qu'il résolve votre désir (votre vœu) ». L'Église Universelle, véritable entreprise religieuse, n'a rien d'altruiste. Comme l'a montré Marion Aubrée, elle a développé " une capacité particulière à recouvrer, grâce à ladite Théologie de la Prospérité, l'argent de ses fidèles. Cette capacité a été à la base de ses succès financiers et de sa fulgurante expansion...» (2003 : 67). André Mary fait, à son sujet, un constat similaire : "L'économie symbolique de la dîme ou les liturgies de dons et d'offrandes permettent incontestablement d'extorquer le maximum d'argent aux fidèles et de tirer profit de situations de désarroi individuel...» (2002: 473).

Entreprise religieuse transnationale (Campos, 1997), l'Église Universelle planifie de manière rigoureuse l'utilisation des médias (radio et TV) et les émissions sont élaborées de façon très soignée (Fonseca, 2004). Dans son ouvrage, Campos décrit la mise en scène finement élaborée des "réunions": une mise en scène conçue autour d'un rituel pensé comme un spectacle (ibid.). Les grandes campagnes annuelles sont décidées et coordonnées, tant du point de vue du contenu, de la scénographie que de la publicité, par les spécialistes du siège de l'Église à Rio de Janeiro au Brésil. Elles sont organisées simultanément partout dans le monde et s'enchaînent sans discontinuer. La campagne précédente n'est pas 
terminée que la suivante pointe le nez, annoncée plus fort encore que la précédente et, comme une nouvelle chance de changement de vie, à ne pas manquer. Ainsi, lorsqu'une campagne vouée à "la victoire sur la fragilité " - rythmée par les distributions hebdomadaires d'huile sainte - dure six semaines et connaitra son dénouement dans une grande cérémonie festive, les pasteurs, voulant maintenir la tension induite par une animation dynamique, censée donner l'image d'une Église en perpétuel mouvement, anticipent et expliquent: "Si demain, vous voulez le succès financier dans votre vie, prenez ce papier que les "ouvriers" sont entrain de distribuer. Inscrivez votre nom et demain lundi à 19 h 30, nous allons vous remettre gratuitement une carte. Vous y trouverez les différentes étapes pour conquérir la prospérité. Dans cette grande campagne, ensemble, semaine après semaine, nous allons les franchir ». La même rigueur se retrouve dans la distribution fréquente et planifiée de multiples petits objets durant les " réunions " (enveloppes, documents, images, petites croix en plastique, fioles d'huile, tissus, cordons, etc.) faisant office de talismans, de reliques (terre d'Israël), d'ex-voto ou d'amulettes, véritables objets transitionnels que le fidèle ramène chez lui, restant ainsi symboliquement relié au temple.

\section{Fréquenter un partenaire qui gagne !}

La modernisation rapide du pays renvoie à un moment particulièrement instable, caractéristique d'une période de croissance économique qui ne se traduit pas automatiquement par une réduction de la précarité pour certains. L'embellie économique conduit aussi une réforme importante de la culture et une augmentation des inégalités sociales, ressenties pour certains comme une forme de souffrance. Il est répondu à l'inquiétude et au doute engendrés par cette situation, et ressentis plus fortement par certains groupes de population, par la promotion d'une identité positive, qualifiée de "gagnante».

L'adhésion à l'Église Universelle en tant qu'entreprise religieuse en pleine croissance répond aux attentes sociales des fidèles et à l'image positive d'euxmêmes qu'ils escomptent acquérir. Par leur implication dans l'édification d'une Église qui se voudrait forte, battante, gagnante, belle, luxueuse et établie au cœur de la cité, ils acquièrent le sentiment diffus d'une revanche sur leur histoire personnelle malheureuse. Dans ce sens, l'expansion rapide de l'Universelle constitue aussi une victoire collective qui rejaillit sur l'identité de chaque membre, lequel considère cette victoire comme sa propre réussite, avant même que de pouvoir accéder concrètement à une vie meilleure. Cette expansion est présentée à la fois comme un miracle et comme un défi qui nécessite l'engagement (financier) de tous les membres. Dans ce cadre, les détracteurs de ce grand projet mobilisateur d'énergie humaine deviennent des ennemis. L'Église Universelle aura alors tôt fait de se présenter en victime persécutée, à l'image des prophètes de la Bible. 
Le diagnostic et le remède aux souffrances multiformes ressenties par les «croyants» (les crentes pour reprendre leurs propres termes) portent, avant tout, sur le rétablissement de l'estime du soi, évitant implicitement de porter la critique sur le champ politique. Au contraire - ce qui facilite peut-être son acceptation par les autorités -, l'Église Universelle du Cap-Vert pousse ses membres à s'approprier du changement comme d'un fait inéluctable de société. Plutôt que de se résigner ou de s'opposer, chacun, là où il est, est poussé à se redéfinir, c'est-à-dire aussi à s'adapter aux mutations sociétales, où le changement et l'insécurité deviennent une composante intrinsèque de la culture capverdienne. Le croyant, renvoyé à lui-même, est invité à entreprendre et à se battre, s'il veut conquérir ce qu'il désire. L'Église pousse ainsi le fidèle, seul responsable de son destin, à conquérir une place digne dans la «nouvelle société ». Dans ce sens, elle développe un discours libéral qu'on peut qualifier d'assimilationniste dans la mesure où l'estime de soi - un des objectifs prônés par l'Église - devrait conduire au bonheur, conçu comme un bien accessible à tous et défini comme l'antidote de la souffrance. Pour l'Église Universelle du Cap-Vert, au-delà de la quête de la santé, certes bien présente dans les propos des fidèles, le bonheur équivaut surtout à accéder à la consommation. Par la promotion d'une morale de la conviction liée à un procès de la responsabilisation, elle met en avant la réussite individuelle qui devrait, dans la foulée, libérer le croyant des anciennes formes de la solidarité, notamment celles liées à la « vieille société capverdienne » toujours louée par de nombreux poètes et chanteurs.

Pierre-Joseph LAURENT

Université catholique de Louvain - Laboratoire d'anthropologie prospective laurent@anso.ucl.ac.be

Claudio FURTADO

Université du Cap-Vert - Vice-recteur à la recherche cfurtado_98@yahoo.com

Almeida R.M., 1996, A universalização do Reino de Deus. Dissertação (Mestrado em Antropologia) Instituto de Filosofia em Ciências Humanas, Universidade de Campinas. Andrade Silva Elisa, 1996, Les îles du Cap-Vert, de la découverte à l'indépendance nationale (1460 à 1975), Paris, L'Harmattan.

-, 2000, Quelques aspects du développement économique, social et politique aux Iles $d u$ Cap-Vert (1975-1999), Paris, UNESCO.

Aubrée Marion, 2001, "Dynamiques comparées de l'Église Universelle du Royaume de Dieu au Brésil et à l'Étranger ", in BASTIAn J.-P., ChAmpion F., Rousselet K., (dirs.) La globalisation du religieux, Paris, L'Harmattan, pp. 113-124.

-, 2003, «Un néo-pentecôtisme brésilien parmi les populations immigrées en Europe de l'Ouest ", in Meintel D. et Leblanc M.-N., (dirs.), Le religieux en mouvement. Anthropologie et sociétés, 27-1, pp. 65-84. 
BASTIAN Jean-Pierre, (dir.), 2001, La modernité religieuse en perspective comparée, Paris, Karthala.

Birman Patricia, 1997, "Males e malef'cios no discurso neopentecostal ", in Birman P., Novaes R., Crespo S., (Organização), O mal à brasileira, Rio de Janeiro, ed. Verj., pp. $62-80$

-, 1998, "Cultes de possession et pentecôtismes au Brésil : passages », Cahiers du Brésil contemporain, 35-36, pp. 185-208.

CAmpos Leonildo Silveira, 1997, Teatro, templo e mercado. Organização e marketing de um empreendimento neopentecostal, Petrópolis-São Paulo-São Bernardo do Campo, Vozes-Simpósio-UMESP.

-, 1999, "A Igreja universal do Reino de Deus, um empreendimento religioso atual e seus modos de expansão (Brasil, África e Europa) ", Lusotopie, pp. 355-367.

-, 2003, « O bem e o mal nas representações de novos pentecostais brasileiras quanto a economia », Caminhos Goiânia, I-2, pp. 33-65.

CARreira Antonio, 2000, Cabo Verde, Formação e extinção de ume sociedade escravocrata (1460-1878), Praia, IPC, p. 208.

CoRTen André, 1995, Le pentecôtisme au Brésil, Paris, Karthala.

Corten André, Dozon Jean-Pierre, Oro ARi Pedro, (dirs.), 2004, Les nouveaux conquérants de la foi. L'Église Universelle du Royaume de Dieu (Brésil), Paris, Karthala.

Certeau Michel de, 1994, La prise de parole et autres écrits politiques, Paris, Seuil.

Documento de estratégia de crescimento e de redução da pobreza (DECRP), Republica de Cabo Verde, Ministério das finanças e do Planeamento, septembre 2004.

Fialho-Costa Livia, 2002, Qu'est-ce qui fait crier les crentes? Émotion, corps et délivrance à l'Église Universelle du Royaume de Dieu (Bahia-Brésil), Thèse de doctorat, Paris, EHESS.

Giumbelli Emerson Alessandro, 2002, O fim da religião. Dilemas da liberdade religiosa no Brasil e na França, São Paulo, Attar.

Gomez Wilson, 1993, «Demônios do fim de século, curas, ofertas e exorcismos na Igreja Universal do Reino de Deus ", Cadernos do CEAS, (Salvador, Ba), 146, pp. 47-63.

Fonseca Alexandre, 2004, "Un empire médiatique ", in Corten A., Dozon J.-P., Oro ARI P., (dirs.), Les nouveaux conquérants de la foi. L'Église Universelle du Royaume de Dieu (Brésil), Paris, Karthala, pp. 211-229.

Freston Paul, 1999, "A Igreja universal do Reino de Deus na Europa ", Lusotopie, pp. 383-403.

-, 2001, "The Transnationalisation of Brazilian Pentecostalism: The Universal Church of the Kingdom of God ", in Corten A., Marshall-Fratani R., (editors), Between Babel and Pentecost. Transnational Pentecostalism in Africa and Latin America, London, Hurst, pp. 196-215.

FurTADO Claudio, 1993, A transformação das estruturas agrárias numa sociedade em mudança-Santiago, Cabo Verde, Praia, Instituto Caboverdiano do Livro e do Disco. Indicateurs PNUD, 2004.

Institut National de Statistique, censo 2000, p. 59.

Latouche Serge, Laurent Pierre-Joseph, Singleton Mike, Servais Olivier, (dirs.), 2004, Les raisons de la ruses, Paris, La Découverte-MAUSS.

LAURENT Pierre-Joseph, 1998, Le don comme ruse. Une association de développement en pays mossi, Paris, Karthala.

-, 2003, Les pentecôtistes du Burkina Faso. Mariage, pouvoir et guérison, Paris, Karthala. 
-, 2005, «The Process of Bricolage Between Mythic Societies and Global Modernity: Conversion to the Assembly of God Faith in Burkina Faso ", Social Compass, 52-3, pp. 309-323.

Macedo Idir, 1996, Orixás, Caboclos e Guias. Deuses ou Demônios? Rio de Janeiro, Gráfica universal. (Production de l’Église Universelle)

Mariano Ricardo, 1995, Neopentecostalismo. Os pentecostais estão mudando, Mémoire de maîtrise présentée à São Paulo-USP.

-, 2004, «Expansão pentecostal no Brasil : o caso da Igreja Universal », Estudos avançados, 18-52, pp. 121-138.

-, 2004, "Le royaume de prospérité de l'Église universelle », in CorTen A., Dozon J.-P., Oro ARI P. (dirs.), Les nouveaux conquérants de la foi. L'Église Universelle du Royaume de Dieu (Brésil), Paris, Karthala, pp. 197-212.

Martin David, 1990, Tongues of fire: the Explosion of Protestantism in Latin América, Oxford, Basil Blackwell.

MARY André, 2000, Le bricolage africain des héros chrétiens, Paris, Cerf.

-, 2001, "Globalisation des pentecôtismes et hybridité du christianisme africain ", in Bastian J.-P., Champion F., Rousselet K., (dirs.), La globalisation du religieux, Paris, L'Harmattan.

-, 2002, «Le pentecôtisme brésilien en Terre africaine. L'universel abstrait du Royaume de Dieu ", Cahiers d'Études africaines, XLII/167, pp. 463-478.

Oro ARI Pedro, 2005, "The politics of the Universal Church and its consequences on religion and politics in Brazil ", trad. Enrique J. Romera, Rev. bras. Ci. Soc. [online], 1, Special Edition [cited 01 February 2006].

Pimentel Fernando da Silva, 2005, "Psiqué nos dominios do demônio, um olhar sobre a relação entre exorcismo e cura num grupo de mulheres fieis da IURD », Revista de Estudos da Religião, 2, pp. 22-24.

PNUD, 2001, Rapport mondial sur le développement humain, annexes, pp. 182-185.

POLANYI Karl, 1983, La grande transformation, Paris, Gallimard.

Ricoeur Paul, 2004, Parcours de la reconnaissance, Paris, Stock.

Rocha Costa Ana, 2003, "La lutte contre le blanchiment de capitaux et le financement du terrorisme ", BCEAO, BEAC, pp. 21-23.

SEN Amartya, 1993, Éthique et Économie, Paris, PUF, coll. « Philosophie morale ».

\section{Résumé}

L'Église Universelle du Royaume de Dieu du Cap-Vert, avec près de 20000 fidèles, sur 475000 habitants (soit $4 \%$ ), connaît, depuis une décennie, un réel succès, dans ce pays-archipel, catholique à plus de $93 \%$. La modernisation rapide du pays (devenu depuis janvier 2008, un Pays de Développement Moyen) renvoie à un moment particulièrement instable. L'embellie économique conduit à une réforme importante de la culture capverdienne et une augmentation des inégalités sociales, ressenties pour certains comme une forme de souffrance. L'Église Universelle répond à l'inquiétude et au doute engendrés par cette situation et ressentis plus fortement par certains groupes de population, par la promotion d'une identité positive, qualifiée de "gagnante". En tant qu'entreprise religieuse, l'Église pousse ses membres à s'approprier du changement comme d'un fait inéluctable de société. Par la promotion d'une morale de la conviction liée à un procès de la responsabilisation, l'Universelle met en avant la 
réussite individuelle qui devrait, dans la foulée, libérer le croyant des anciennes formes de la solidarité, notamment celles liées à la "vieille société capverdienne " et toujours louée par de nombreux poètes et chanteurs.

Mots-clés : Cap-Vert, Église Universelle, développement.

\section{Abstract}

The Universal Church of the Realm of the God of Cape Verde, with about 20000 believers, on 475000 inhabitants (around 4\%), has been, for a decade, a real success, in this country-archipelago which counts about $93 \%$ Catholics. The fast modernization of the country (since January 2008, a Country of Average Development) sends at a particularly unstable moment. The economics' taking off leads to an important reform of the capeverdean culture and an increase in the social disparities, understood for some as way of suffering. The Universal Church answers the anxiety and the doubt engendered by this situation, and more strongly felt by certain groups of population, by the promotion of a positive identity, qualified as "winning".

As religious company, the Church urges its members to appropriate of the change as the inevitable fact of society. By the promotion of a morality of the conviction related to a lawsuit of the empowerment, the Universal advances the individual success which should, in the stride, release the believer of the ancient forms of the solidarity, notably those connected to the "old Capeverdean society" and always praised by numerous poets and singers.

Key words: Cape Verde, Universal Church, development.

\section{Resumen}

La Iglesia Universal del Reino de Dios de Cabo Verde, con cerca de 20000 creyentes, sobre 475000 habitantes (el $4 \%$ ), conoce, desde hace una década, un éxito real, en éste país-archipiélago, católico a más del $93 \%$. La modernización rápida del país (convertido en un País de Desarrollo Medio, desde enero de 2008) da como resultado un momento particularmente inestable. El realce económico conduce a una importante reforma de la cultura caboverdiana y a un aumento de las desigualdades sociales, sentidas par algunos como una forma de sufrimiento. La Iglesia Universal responde a la inquietud y a la duda engendrada por ésta situación y sentido con mayor fuerza por ciertos grupos de población, mediante la promoción de una identidad positiva, cualificada de "ganadora".

Como empresa religiosa, la Iglesia incita a sus miembros a apropiarse del cambio como de un hecho ineludible de sociedad. Mediante la promoción de una moral de la convicción vinculada a la responsabilización, la Universal pone por delante el éxito individual que debería, en la corriente, liberar al creyente de las antiguas formas de la solidaridad, particularmente las vinculadas a la "vieja sociedad caboverdianas" $y$ siempre ensalzada por numerosos poetas y cantantes.

Palabras clave: Cabo Verde, Iglesia Universal, desarrollo. 
